\title{
Keyword Index
}

Volume 51998

421678

1801678

Aberrant cell cycle 60

Abrin 231

Actin cytoskeleton 921

Alphavirus 50

Altered in vivo differentiation 765

Alzheimer's disease 858

AML1 short isoform 765

Anchorage independent growth 440

Anticancer drugs 735, 1062

Antiphospholipid autoantibodies 563

Antisense 440

Antisense RNA 20

APC 206

Apoptosis 4, 11, 20, 29, 38, 50, 60, 67, $77,87,107,116,132,141,148,156$, $163,172,183,191,200,206,214$, 231, 241, 262, 271, 289, 298, 307, $321,346,365,372,390,432,440$, 450, 469, 480, 506, 517, 529, 551, $577,584,592,615,623,653,660$, $669,687,702,710,735,743,758$, $774,785,805,823,838,847,858$, 867, 876, 884, 894, 901, 921, 946, 953, 963, 973, 984, 1004, 1017, 1034, 1062

Apoptosis calpastatin 1028

Apoptosis induction 426

Ara-C 584

Argos 262

ATP 191

Atresia 38, 67

Autoantibodies 183, 222

Autoimmune lymphoproliferative syndrome 751

Autoimmunity 563

Axon arborization 816

Axon elongation 816

$\beta$-Amyloid precursor protein 858

$\beta$-APP deficient mice 858

B-myb 401

BAX 67

Bax 506, 687, 805, 901, 911

BCL-2 67, 107, 901

Bcl-2 77, 298, 432, 601, 660, 687, 816, 901, 911, 1034, 1062

Bcl-2 protein family expression 408

BCL-X 67

Bcl-x 805, 901

BCR-ABL 710

Bcr-Abl 298
bFGF 911

Bile acids 623

Blastodermal cells 529

BP53.12 678

Brain 116, 321, 357

Brain tumors 884

Breast carcinoma 307

c-myc 381, 758

c-Myc 141

C3 720

$C A D$ gene amplifcation 984

Cadherins 1042

Calpain 1028, 1051

Canale-Smith syndrome 751

Cancer chemotherapy 506

Caspase 67, 271, 506, 569, 660, 729, 823, 838, 973, 1017, 1028

Caspase family 1034

Caspase inhibitors 432

Caspase-3 (CPP32) 289

Caspase-3 469, 592, 911, 1004

Caspases 107, 298, 432, 488, 637, 646, 735, 847, 894, 953, 1042, 1051

CCRF-CEM 687

$\mathrm{CD}^{+} \mathrm{T}$ cell depletion 222

$\mathrm{CD}^{+}$cells 231

CD95 (APO-1/Fas) 222, 884

CD95 (Fas, APO-1) 271, 894

CD95 200, 735

CD95/APO-1/Fas 87

CD95L 735

Cdc2 132

Cdc25A 758

Cdks 132

CED-3 838

Cell adhesion 921

Cell cycle 132, 687, 940

Cell death 107, 163, 191, 298, 365, $416,432,637,838$

Cell death genes, developmental expression 529

Cell proliferation 973

Cell regulation 720

Cell spreading 921

Cell surface molecules 231

Cell-growth 20

Central nervous system 357

Ceramide 29, 172, 241, 346, 357, 785, 876

Ceramides 381

Cerebral energy metabolism 321

c-fos 858
Chemotherapy 876

Chick embryo 60

Children 222

Chondrocyte 60

CHP-100 785

CIF 469

Cisplatin 601

Cisplatin-resistance 390

Cloning 838

Clonogenic 107

Clonogenicity 96, 984

CML 710

CNF1 720, 921

Colicin 372

Colon carcinoma cells 450

Colorectal cancer 480

Compound eye 262

CPP-32 592

CPP32 67, 241

Crypt 702

Cyclins 132

Cyclophosphamide 592

Cytochrome c 469, 660

Cytochrome c 646, 953

Cytokine 569

Cytolytic lymphocyte 488

Cytopathic effect 653

Cytotoxicity 191, 231

dATP 469

Dermal alterations 792

Dementia 832

Development 156, 930

Diabetes mellitus 461

Diap2 930

Dictyostelium 416

Diethylmaleate 313

Differential display 615

Differential gene expression 615

Differential screening 116

Differentiation 77, 251, 401

DNA cleavage 38

DNA damage 623

DNA fragmentation 517, 592, 702

DNA ladder 592

DNases 251

D01 678

DO7 678

Dorsal root ganglion 876

Drosophila 262, 569, 930

Drug resistance 694, 1062

Drugs 884

E2F 401 
Early growth response- 1 transcription factor 96

Egr-1 response element 96

Endonuclease 390, 1017

Endonucleases 517

Enhanced apoptosis 765

Epilepsy 867

ES cells induced teratocarcinomas 765

Etoposide resistance 529

Evolution 416

Excitotoxicity 847, 858, 867

Exocrinopathy 743

Extracellular matrix (ECM) 601

Eye 156

Fas/apo-1 mutation 751

Fas ligand 4

Fas signalling 450

Fas 200, 241, 289, 426, 480, 729, 743, 963

Fas-ligand 426

FGF 774

Fodrin 1051

Follicle 38

Follicular dendritic cells 497

$\gamma$-irradiation 884

G1 arrest 148

Gal4/UAS 930

Gamma irradiation 687

Gene expression 11, 116, 450

Genome cleavage 251

Genomic instability 984

Giant cell 669

Glioma 894

Glucocorticoid 1034

Glucosylceramide 785

Granulosa cell 38, 67

Granzyme 488

Grim 930

Growth arrest 381

Growth plate 60

Heart 592

Heat and cold shock 529

HIV 222

HT1080 fibrosarcoma cells 96

Human cancers 1062

Human leukemia 687

Hyperthermia 592

Hypoxia 141

IAP 569

ICAM-1 497

ICE 823, 838

$\mathrm{ICH}-167$

IGF-1 911

Immunoblotting 678

Immunofluorescence 678
Immunostaining 1004

Inflammation 191, 563

Influenza virus 426

Insertional mutagenesis 416

Insulin 901

Insulin-like growth factors 901

Insulin secretion 461

Interferon 313

Interleukin-1 461

Intracellular $\mathrm{pH} 214$

In vitro 601

In vitro embryo culture 592

Irradiation 172, 584, 669

Ischemia/reperfusion injury 271

ISEL+ 702

K562 710

Kainic acid 858, 867

Kidney 601

KYO-1 710

L1210 leukemia cells 390

LBP-p40 20

Lectin 615

Lens cells 251

Lewy bodies 832

Lung carcinoma 148

Lymphocyte 200

Lymphocytes 77

Lymphoid organization 497

Lymphoma 506, 973

Malonate 847

Melanoma cell lines 946

Membrane 372

Membranes 551

Microinjection 660

Milk proteins 440

Mistletoe lectins 231

Mitochondria 646, 694, 805

Mitochondrial discontinuities 953

Mitochondrial permeability transition 214

Mitochondrial ultracondensation 953

Mouse hepatoma cells 87

Mouse 669

MRS 321

Murine development 408

Mycoplasma hyorhinis 517

Myeloid leukemia 172

Necrosis 183,432

Nedd2 847

Nephrotoxicity 601

Nerve growth factor 50, 346

Neuroblastoma 401

Neuroepithelioma cells 785

Neuroepithelium 592

Neurodegeneration 805, 867

Neurofilaments 832
Neuron 50, 1004

Neuronal cell death 816

Neuronal cultures 858

Neuronal death 805

Neurotrophin 365

Neurotrophins 346

Newborn 321

NDGA 894

NF- $\kappa$ B 623, 963

Nitric oxide 461, 911

Nuclear apoptosis 251

Nuclear localization 488

Nuclear proteins 183

Nucleases 38

Oct-1 774

Oncogenes 298

Organotypic coculture 816

Ovary 38, 67

Overexpression 440

Oxidative stress 623,940

p21 390, 1062

p21-activated kinase 637

p21 waf1/cip1 940

p21 WAF1/CIP1/Sdi1 381

21/WAF1 687

p27 1062

p35 569

p5367, 87, 141, 148, 156, 390, 669,

$678,687,984$

p53 phosphorylation 584

Pancreatic islet 461

Parkinson's disease 832

PARP 592, 623, 1042

Pathological cell death 823

Perforin 488

Peripheral neuropathy 876

Permeability transition 646

Phagocytosis 551, 563

Phosphatases 940

Phosphatidylserine externalization 426

Phosphatidylserine 551

Phosphorylation 96

Photoaging 792

PHPV 156

PI-3K 289

Plasminogen activator inhibitor 163

Platelet activating factor 867

Porcine 601

Postimplantation mouse embryo 592

POU domain 774

pRb 132

Primary culture 50

Programmed cell death 50, 262, 313, 569, 615, 702, 729, 1004

Prohibitin 615

Protease 1017

Proteases 38, 183 
Proteasome inhibitors 577, 1062

Protein synthesis inhibition 231

Proteolysis 29

Purinergic receptors 191

\section{RACK 615}

Radiation 77, 141, 1051

RAR 11

Ras 262, 729

ras oncogene 984

$\mathrm{Rb} 206$

$\mathrm{Rb} 2 / \mathrm{p} 130401$

Receptor 365

Receptors 551

Rel 963

Resistance 172

Reticular dendritic cells 497

Retinal ganglion cells 816

Retinoblastoma family 401

Retinoic acid 4, 11

Retinoic acid receptor 11

Retinoids 973

Retroviruses 440

Rho 720, 921

Ribozyme 20

Ricin 231

RING finger 569

RXR 11

Salivary gland 743
Semliki Forest virus 50

Serpin 163

Signal transduction 4, 637

Signalling 29

Sindbis Virus 577

Sjögrens syndrome 743

Skin equivalent 792

Skin morphogenesis 792

Small intestine 702

Sodium arsenite 592

Sodium butyrate 307,480

Sodium deoxycholate 623

Soluble CD95 222

Spermatogenesis 669

Spermatogonial stem cell 669

Sphingomyelinase 241

Spleen 497

Spontaneous in vitro apoptosis 751

Staurosporine 313

Synuclein 832

T lymphocytes 4, 29

T-cell 200

Teeth 408

Telencephalon 901

Teratogens 592

Therapeutic window 847

Thymus 497

Tissue transglutaminase 4,11

TNF 241, 289, 758
TNF- $\alpha 963$

Transactivation 96

Trypanosoma brucei rhodesiense 615

Tumor necrosis factor receptors 357

Tumor necrosis factor 87, 163, 346

Tumor suppressors 984

Tumorigenicity 440, 694

U937 694

Ubiquitin 307

UV 1042

UV-C response 96

UVA radiation 792

Valinomycin 214

Villus 702

Viral persistence 653

Virus 653

Viruses 313

Vitamin D 116

Vitamin $D_{3} 946$

Vitamin $D_{3}$ analogues 946

Wortmannin 289

Xenopus 774

YVAD.CMK 206

Z-VAD.FMK 206 